Review began 11/03/2021 Review ended 11/22/2021 Published 11/25/2021

๑) Copyright 2021

Ried et al. This is an open access article distributed under the terms of the Creative Commons Attribution License CC-BY 4.0., which permits unrestricted use, distribution, and reproduction in any medium, provided the original author and source are credited.

\section{Therapies to Prevent Progression of COVID-19, Including Hydroxychloroquine, Azithromycin, Zinc, and Vitamin D3 With or Without Intravenous Vitamin C: An International, Multicenter, Randomized Trial}

Karin Ried ${ }^{1,2,3}$, Taufiq BinJemain ${ }^{4}$, Avni Sali ${ }^{5}$

1. NIIM Research, National Institute of Integrative Medicine, Melbourne, AUS 2. Health and Nutrition, Torrens University, Melbourne, AUS 3. Discipline of General Practice, The University of Adelaide, Adelaide, AUS 4. Gold Coast Clinic, National Institute of Integrative Medicine, Gold Coast, AUS 5. NIIM Clinic, National Institute of Integrative Medicine, Melbourne, AUS

Corresponding author: Karin Ried, karinried@niim.com.au

\title{
Abstract
}

\section{Background}

COVID-19 is a global pandemic. Treatment with hydroxychloroquine (HCQ), zinc, and azithromycin (AZM), also known as the Zelenko protocol, and treatment with intravenous (IV) vitamin C (IVC) have shown encouraging results in a large number of trials worldwide. In addition, vitamin D levels are an important indicator of the severity of symptoms in patients with COVID-19.

\section{Objectives}

Our multicenter, randomized, open-label study aimed to assess the effectiveness of HCQ, AZM, and zinc with or without IVC in hospitalized patients with COVID-19 in reducing symptom severity and duration and preventing death.

\section{Methods}

Hospitalized patients with COVID-19 in seven participating hospitals in Turkey were screened for eligibility and randomly allocated to receive either HCQ, AZM, and zinc (group 1) or HCQ, AZM, zinc plus IV vitamin C treatment (group 2) for 14 days. The patients also received nontherapeutic levels of vitamin D3.

The trial is registered on the Australian and New Zealand Clinical Trial Registry ACTRN12620000557932 and has been approved by the Australian Therapeutic Goods Administration (TGA).

\section{Results}

A total of 237 hospitalized patients with COVID-19 aged 22-99 years (mean: $63.3 \pm 15.7$ years) were enrolled in the study. Almost all patients were vitamin D deficient (97\%), 55\% were severely vitamin D deficient $(<25$ $\mathrm{nmol} / \mathrm{L})$ and $42 \%$ were vitamin D deficient ( $<50 \mathrm{nmol} / \mathrm{L}) ; 3 \%$ had insufficient vitamin D levels $(<75 \mathrm{nmol} / \mathrm{L})$, and none had optimal vitamin D levels.

Of the patients, $73 \%$ had comorbidities, including diabetes (35\%), heart disease (36\%), and lung disease (34\%).

All but one patient $(99.6 \%$; $=236 / 237)$ treated with HCQ, AZM, and zinc with or without high-dose IV vitamin C (IVC) fully recovered. Additional IVC therapy contributed significantly to a quicker recovery (15 days versus 45 days until discharge; $\mathrm{p}=0.0069)$.

Side effects such as diarrhea, nausea, and vomiting, reported by $15 \%-27 \%$ of the patients, were mild to moderate and transient. No cardiac side effects were observed.

Low vitamin D levels were significantly correlated with a higher probability of admission to the intensive care unit (ICU) and longer hospital stay.

Sadly, one 70-year-old female patient with heart and lung disease died after 17 days in ICU and 22 days in the hospital. Her vitamin D level was $6 \mathrm{nmol} / \mathrm{L}$ on admission (i.e., severely deficient).

\section{Conclusions}

Our study suggests that the treatment protocol of HCQ, AZM, and zinc with or without vitamin C is safe and 
effective in the treatment of COVID-19, with high dose IV vitamin C leading to a significantly quicker recovery.

Importantly, our study confirms vitamin D deficiency to be a high-risk factor of severe COVID-19 disease and hospitalization, with $97 \%$ of our study's patient cohort being vitamin D deficient, $55 \%$ of these being severely vitamin D deficient, and none had optimal levels.

Future trials are warranted to evaluate the treatment with a combination of high-dose vitamin D3 in addition to HCQ, AZM, and zinc and high-dose intravenous vitamin C.

Categories: Infectious Disease, Therapeutics, Integrative/Complementary Medicine Keywords: vitamin d, intravenous vitamin c, zinc, hydroxychloroquine, covid-19 treatment, covid-19

\section{Introduction}

The severe acute respiratory syndrome coronavirus 2 (SARS-CoV-2), or COVID-19, has affected millions of people worldwide. COVID-19 was first reported by the World Health Organization in December 2019 and was declared a worldwide pandemic in March 2020. Exploring therapies potentially of benefit for COVID-19 has been a public health emergency.

SARS-CoV-2 enters cells by binding to the ACE2 receptor. Higher blood levels of ACE2 reflect shedding from the myocardium and pulmonary epithelium and identify patients who are vulnerable to the development of life-threatening complications.

Early in the pandemic, the combination of hydroxychloroquine (HCQ), azithromycin (AZM), and zinc, also known as the Zelenko protocol, had shown great promise in the treatment of COVID-19 [1,2].

In vitro, chloroquine increases the endosomal $\mathrm{pH}$ required for the virus to fuse with cells and interferes with the glycosylation of SARS-CoV-2 cell receptors, thereby blocking viral infection [3,4]. Investigators performed a time-of-addition assay, which showed that chloroquine is effective at both the entry and postentry stages of the SARS-CoV-2 infection in cells. Hydroxychloroquine has greater in vitro potency than chloroquine against SARS-CoV-2 and, because of its enhanced safety profile, can be given at higher doses than chloroquine [5].

As of October 2021, a meta-analysis of more than 290 worldwide trials involving more than 412,000 patients found that HCQ significantly reduced morbidity and mortality in patients with COVID-19. Specifically, when HCQ is used in early treatment, a meta-analysis of 32 studies involving more than 54,600 patients suggested HCQ to improve symptoms and prevent death by $64 \%-75 \%$ (all early treatment studies $(n=32): R R, 0.36$ $(0.29-0.46), p<0.0001$; early treatment studies reporting mortality $(n=13): R R, 0.25(0.16-0.40), p<0.0001)$ [6].

Azithromycin is a macrolide antibiotic that has been found to inhibit the viral tropism and replication of Zika and Ebola viruses $[7,8]$. An in vitro study has shown the activity of azithromycin (AZM) in combination with hydroxychloroquine (HCQ) against SARS-CoV-2 [9].

In addition, the effectiveness of this combination therapy of HCQ and AZM, when used early, as was demonstrated in a clinical study involving 83 patients in Turkey, reduced recovery time and shortened hospital length of stay [10].

In therapeutic doses, $\mathrm{HCQ}$ has a high safety profile and works as a zinc ionophore, enabling zinc to enter a virus-infected cell, increasing intracellular zinc concentrations [11].

Zinc itself has antiviral properties, boosting both innate and humoral immunity [12]. High intracellular concentrations of zinc are essential to inhibit viral replication and proliferation, including coronavirus RNAdependent RNA polymerase activity [13].

The Zelenko COVID-19 treatment protocol consists of the following triple therapy for five consecutive days in addition to standard supportive care: zinc sulfate (220 mg capsule once daily, containing $50 \mathrm{mg}$ elemental zinc), HCQ (200 mg twice daily), and AZM (500 mg once daily) [2].

In addition, intravenous vitamin C (IVC) has known immune-stimulating and antiviral properties [14] and had shown promise as a treatment for acute respiratory syndrome and pneumonia [15]. Recent studies reported on the benefits of IVC therapy for COVID-19 [16,17].

Furthermore, a large number of studies $(n>200$ ) have demonstrated low vitamin $D$ levels to be a risk factor for the severity of COVID-19 symptoms and hospitalization [18-20]. 
Adequate vitamin D levels are of great importance in the prevention of respiratory infections, as vitamin D protects against pathogens including viruses via the innate and adaptive immune systems, involving white blood cells and T-cells [21].

In our study, we aimed to assess the optimal treatment protocol for hospitals to consider in their treatment for patients with COVID-19, in order to reduce the severity and duration of symptoms and save lives. Patients presenting at hospitals with COVID-19 symptoms were randomly allocated to the Zelenko protocol $(\mathrm{HCQ}+\mathrm{AZM}+$ zinc) or the Zelenko protocol plus IV vitamin C.

All enrolled patients also received supplementation of 5000 IU/day of vitamin D3, an adequate dose if levels of vitamin D are insufficient (51-75 nmol/L); however, this dose is considered inadequate for vitamin D deficiency $(<50 \mathrm{nmol} / \mathrm{L})$.

\section{Materials And Methods \\ Trial design and participants}

Our study is an international, multicenter, open-label, randomized controlled trial evaluating the efficacy and safety of therapies with hydroxychloroquine (HCQ), azithromycin (AZM), zinc, and vitamin D3 alone (group 1) or HCQ + AZM + zinc in combination with IV vitamin C (group 2) in hospitalized patients with COVID-19. For stage 1 of the trial, we aimed to recruit 200 patients.

The trial was conducted in Australia and Turkey between January and June 2021. Stage 1 of the trial took place primarily in Turkey and involved seven participating hospitals in Eskisehir, Elazig, Istanbul, Erzincan, and Izmir.

The trial was approved by the National Health and Medical Research Council (NHMRC)-endorsed National Institute of Integrative Medicine (NIIM) Human Research Ethics Committee in Australia, the Turkish Ethics Committees at the Ministry of Health in Turkey, and participating hospitals.

The trial is registered on the Australian and New Zealand Clinical Trial Registry ACTRN12620000557932 and has been approved by the Australian Therapeutic Goods Administration (TGA).

All eligible participants provided electronic written informed consent.

\section{Inclusion criteria}

The inclusion criteria were as follows: (1) age $\geqslant 18$ years, (2) informed written consent, and (3) diagnosis of active symptomatic COVID-19 confirmed by polymerase chain reaction (PCR) testing via nasal and/or oral swab at the time of enrolment for quantitative PCR assessment.

\section{Exclusion criteria}

The exclusion criteria were as follows: (1) known G-6-PDH deficiency; (2) contraindication to hydroxychloroquine, azithromycin, or vitamin C, allergy to study

interventions, epilepsy, serious hearing or visual problems, advanced liver disease, history of severe depression, calcium oxalate stones, and pregnant or lactating women; (3) already receiving hydroxychloroquine, azithromycin, vitamin C >3 g daily, or an experimental antiviral; (4) history of fever (e.g., night sweats and chills) and/or acute respiratory infection (e.g., cough, shortness of breath, and sore throat) of more than seven days' duration; (5) calculated creatinine clearance of $<30 \mathrm{~mL} / \mathrm{minute}$; (6) baseline electrocardiogram (ECG) showing QTc $\geqslant 470$ for males and QTc $\geqslant 480$ for females; and (7) receipt of a drug known to increase QTc, such as quetiapine, amiodarone, and sotalol.

\section{Intervention}

Group 1 received $\mathrm{HCQ}+$ zinc + AZM + vitamin D3, whereas group 2 received vitamin $\mathrm{C}+$ group 1 interventions. Hydroxychloroquine (HCQ) was given as $400 \mathrm{mg}$ peroral (PO) once a day for one day, followed by $200 \mathrm{mg}$ once a day for six days. Azithromycin (AZM) was given as $500 \mathrm{mg}$ PO on day 1, followed by $250 \mathrm{mg}$ PO once daily for four days. Zinc citrate was given as $30 \mathrm{mg}$ elemental zinc PO daily for 14 days. Vitamin D3 was given as 5,000 IU PO daily for 14 days. IV vitamin C (sodium ascorbate) was given as $50 \mathrm{mg} / \mathrm{kg}$ every six hours on day 1 , followed by $100 \mathrm{mg} / \mathrm{kg}$ every six hours (four times daily, $400 \mathrm{mg} / \mathrm{kg} / \mathrm{day}$ ) for seven days (average: $28 \mathrm{~g}$ /day; maximum dose: $50 \mathrm{~g} / 24$ hours for those weighing more than $125 \mathrm{~kg}$ ).

\section{Data collection}

Project management and data collection were carried out by appointed teams at the participating sites.

The participants' gender, age, disease severity, comorbidities (smoking, diabetes, heart disease, lung disease, and immunosuppression), other medications, and trial outcomes were entered into an electronic 


\section{Cureus}

online database using Microsoft Forms questionnaires.

\section{Outcomes}

Primary Outcome

The primary outcome was mortality or need for invasive mechanical ventilation at any time in the first 15

days from enrolment.

Secondary Efficacy Outcomes

The secondary efficacy outcomes (measured at both 15 and 45 days from enrolment) are mortality, invasive mechanical ventilation, need for humidified high-flow oxygen, admission to the intensive care unit (ICU), days in the hospital, days in the ICU, renal replacement therapy, and extracorporeal support.

The secondary efficacy outcomes also include the World Health Organization (WHO) Master Protocol ordinal score at day 15 as follows: (1) not hospitalized, no limitations on activities; (2) not hospitalized, limitation on activities; (3) hospitalized, not requiring supplemental oxygen; (4) hospitalized, requiring supplemental oxygen; (5) hospitalized, on noninvasive ventilation or high-flow oxygen devices; (6) hospitalized, on invasive mechanical ventilation or ECMO; and (7) death.

Secondary Safety Outcomes

The secondary safety outcomes are QTc prolongation ( $>500 \mathrm{~ms}) 24$ hours following the initial dose of study drugs, serious ventricular arrhythmia (including ventricular fibrillation) or sudden unexpected death in the hospital, and any of the following adverse events in the first 10 days from enrolment: diarrhea, grade 2 or greater; nausea, grade 2 or greater; and vomiting, grade 2 or greater (Appendices).

\section{Adaptive design features}

The study was overseen by the Steering Committee consisting of chief investigators (TB, KR, and AS) and investigators at recruited sites. Independent Data Safety Monitoring Committees (DSMC) at participating hospitals monitored the progress and safety of the trial treatment and were to make recommendations on whether to continue, modify, or stop the trial for safety or ethical reasons.

\section{Sample size calculation}

In stage 1 , the sample size required is $\mathrm{n}=100$ in each intervention arm in order to have a statistical power of $80 \%$ to detect a relative risk reduction (RRR) of $30 \%$ in the proportion progressing to mechanical ventilation or death, compared with standard care, and assuming a standard-of-care risk of progression of 30\%. Since the participants were hospitalized, we assumed minimal $(<1 \%)$ loss to follow-up. The total sample size was n $=200$.

Analyses were performed using IBM SPSS version 26 . Statistical significance was set at $\mathrm{p}<0.05$. The primary analysis of efficacy was conducted under the intention-to-treat principle; all randomized participants were included in the analyses. Descriptive analysis was conducted on all variables. Any variable differences between groups were included in analyses as covariates. Differences between the groups and comparison of continuous outcome variables were analyzed using Student's t-test or analysis of covariance (ANCOVA) and chi-square analysis for dichotomous variables or Mann-Whitney U-tests for ranking variables. Correlations between variables were assessed using Pearson's correlation coefficient.

\section{Results}

Seven hospital sites in Turkey participated in the multicenter trial (Figure 1). 


\section{Cureus}

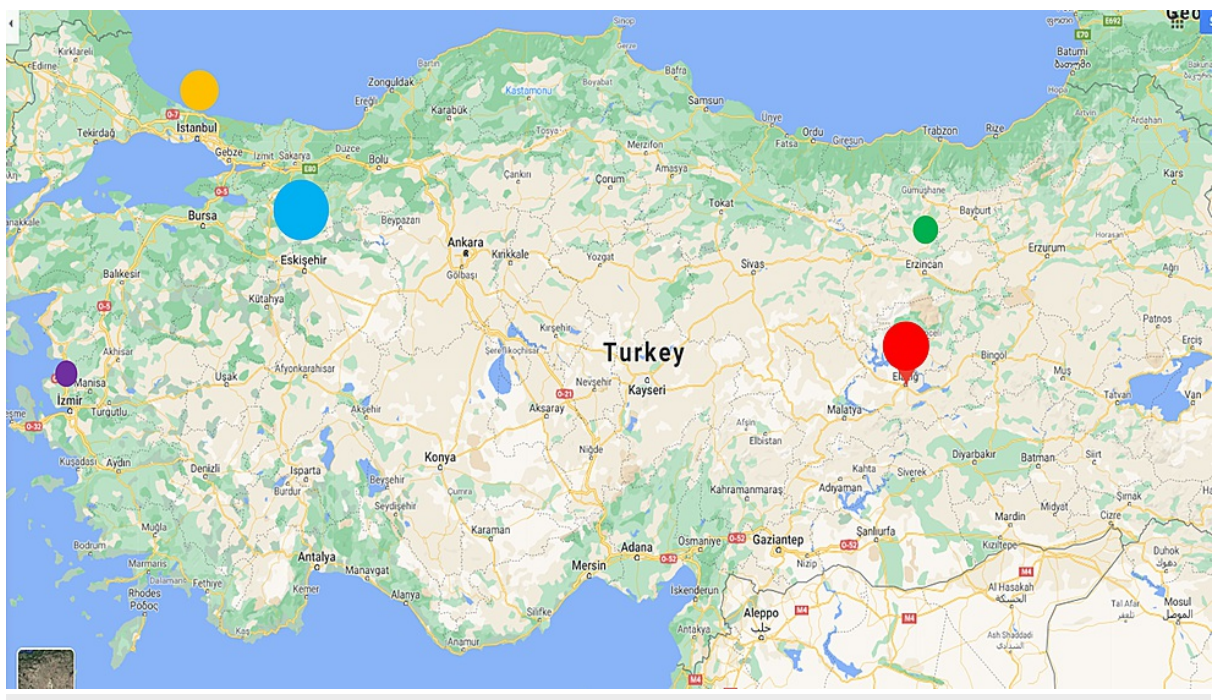

FIGURE 1: Participating hospital sites in Turkey

\section{Participants}

In total, 237 hospitalized patients were enrolled in the study in Turkey. Table 1 outlines the number (\%) of patients enrolled by hospital site.

\begin{tabular}{|l|l|l|}
\hline Hospital site & Color circle on the map (Figure 1) & Number of patients enrolled \\
\hline Eskisehir (ESOGU) & Blue & 138 \\
\hline Eskisehir (City Hospital) & Red & 26 \\
\hline Elazig & Yellow & 67 \\
\hline Istanbul & Green & 1 \\
\hline Istanbul (University) & Purple & 24 \\
\hline Erzincan & & 13 \\
\hline Izmir & & $25 \%$ \\
\hline
\end{tabular}

TABLE 1: Number (\%) of patients enrolled by hospital site

The average age of the patients enrolled was $63.3 \pm 15.7$ years, ranging from 22 to 99 years. Half of the patients were male and half were female. All findings were independent of age and gender (Table 2).

Out of the enrolled patients, 96\% tested positive by the COVID-19 PCR test; those who tested negative had severe respiratory symptoms, such as cough and difficulty breathing $(9 / 10)$, or had tested positive with a COVID-19 antibody test (1/10).

A small number of patients had recently received a vaccination: $2 / 230$ (3\%) reported to have received a flu vaccination and 5/232 (2\%) had received a COVID-19 vaccination at the time of the study.

Of the participants, $73 \%$ had comorbidities, including diabetes (35\%), heart disease (36\%), lung disease (34\%) or were heavy smokers $(41 \%)$, cancer $(10 \%)$, or autoimmune disease (3\%) (Table 2 ).

Almost all hospitalized patients with COVID-19 enrolled in the study were vitamin D deficient (97\%), 55\% were severely vitamin D deficient $(<25 \mathrm{nmol} / \mathrm{L})$ and $42 \%$ were vitamin D deficient $(<50 \mathrm{nmol} / \mathrm{L}) ; 3 \%$ had insufficient vitamin D levels ( $<75 \mathrm{nmol} / \mathrm{L}$ ), and none had optimal vitamin D levels (Table 2, Figure 2). 


\section{Cureus}

\begin{tabular}{|c|c|c|}
\hline $\begin{array}{l}\text { Hospitalized } \\
\text { patients/outpatients }\end{array}$ & $237(90 \%) / 29(10 \%)$ & Only hospitalized patients were included in the analysis. \\
\hline Gender (M/F) & $119(50 \%) / 118(50 \%)$ & \\
\hline Age (years) & $\begin{array}{l}63.3 \pm 15.7(\text { range: } 22-99) \\
\text { years }\end{array}$ & \\
\hline $\begin{array}{l}\text { COVID-PCR } \\
\text { (positive/negative) }\end{array}$ & $227(96 \%) / 10(4 \%)$ & $\begin{array}{l}\text { 9/10 with negative PCR had severe respiratory symptoms (cough/difficulty } \\
\text { breathing). }\end{array}$ \\
\hline Flu/COVID-19 vaccination & $7 / 230,5 / 232$ & \\
\hline Days unwell at enrolment & $4 \pm 3$ (range: $1-30)$ & \\
\hline Vitamin D level (nmol/L) & $24.1 \pm 9.2$ (range: $2-64$ ) & \\
\hline \multicolumn{3}{|l|}{$\begin{array}{l}\text { Vitamin D categories (Figure } \\
\text { 2) }\end{array}$} \\
\hline $\begin{array}{l}\text { Severely deficient }(<25 \\
\mathrm{nmol} / \mathrm{L})\end{array}$ & $131(55 \%)$ & The majority were deficient in vitamin $\mathrm{D}$; half were severely deficient. \\
\hline Deficient (25-50 nmol/L) & $99(42 \%)$ & \\
\hline Insufficient (51-75 nmol/L) & $7(3 \%)$ & \\
\hline Optimal (>75 nmol/L) & None & \\
\hline Zinc (umol/L) & $17.6 \pm 4.4$ (range: $5-34)$ & \\
\hline Deficient (<14 umol/L) & $49(21 \%)$ & \\
\hline Normal (14-23 umol/L) & $169(71 \%)$ & \\
\hline Excess (>23 umol/L) & $19(8 \%)$ & \\
\hline $\mathrm{Mg}(\mathrm{mg} / \mathrm{dL})$ & $1.88 \pm 0.27$ & \\
\hline Deficient (<1.7 mg/dL) & $48(20 \%)$ & \\
\hline Normal (1.7-2.2 mg/dL) & $177(75 \%)$ & \\
\hline Excess (>2.2 mg/dL) & $12(5 \%)$ & \\
\hline Variable & & $\mathrm{N}(\%)$ \\
\hline Comorbidities (1+) & & $172(73 \%)$ \\
\hline Diabetes & & $82(35 \%)$ \\
\hline Heart disease & & $86(36 \%)$ \\
\hline Lung disease & & $56(34 \%)$ \\
\hline Heavy smoker & & $98(41 \%)$ \\
\hline Cancer & & $10(4 \%)$ \\
\hline Autoimmune disease & & $7(3 \%)$ \\
\hline \multicolumn{3}{|c|}{ Biomarkers (cardiopulmonary - abnormal) } \\
\hline Prothrombin & & $28(12 \%)$ \\
\hline D-dimer & & $40(17 \%)$ \\
\hline Fibrin + FDP & & $37(16 \%)$ \\
\hline Troponin & & $46(19 \%)$ \\
\hline BNP & & $32(14 \%)$ \\
\hline
\end{tabular}

\section{TABLE 2: Participant characteristics}

M, male; f, female; N, number; SD, standard deviation; nmol/L, nanomole per liter; umol/L, micromole per liter; mg/dL, milligram per deciliter; BNP, brain 


\section{Cureus}

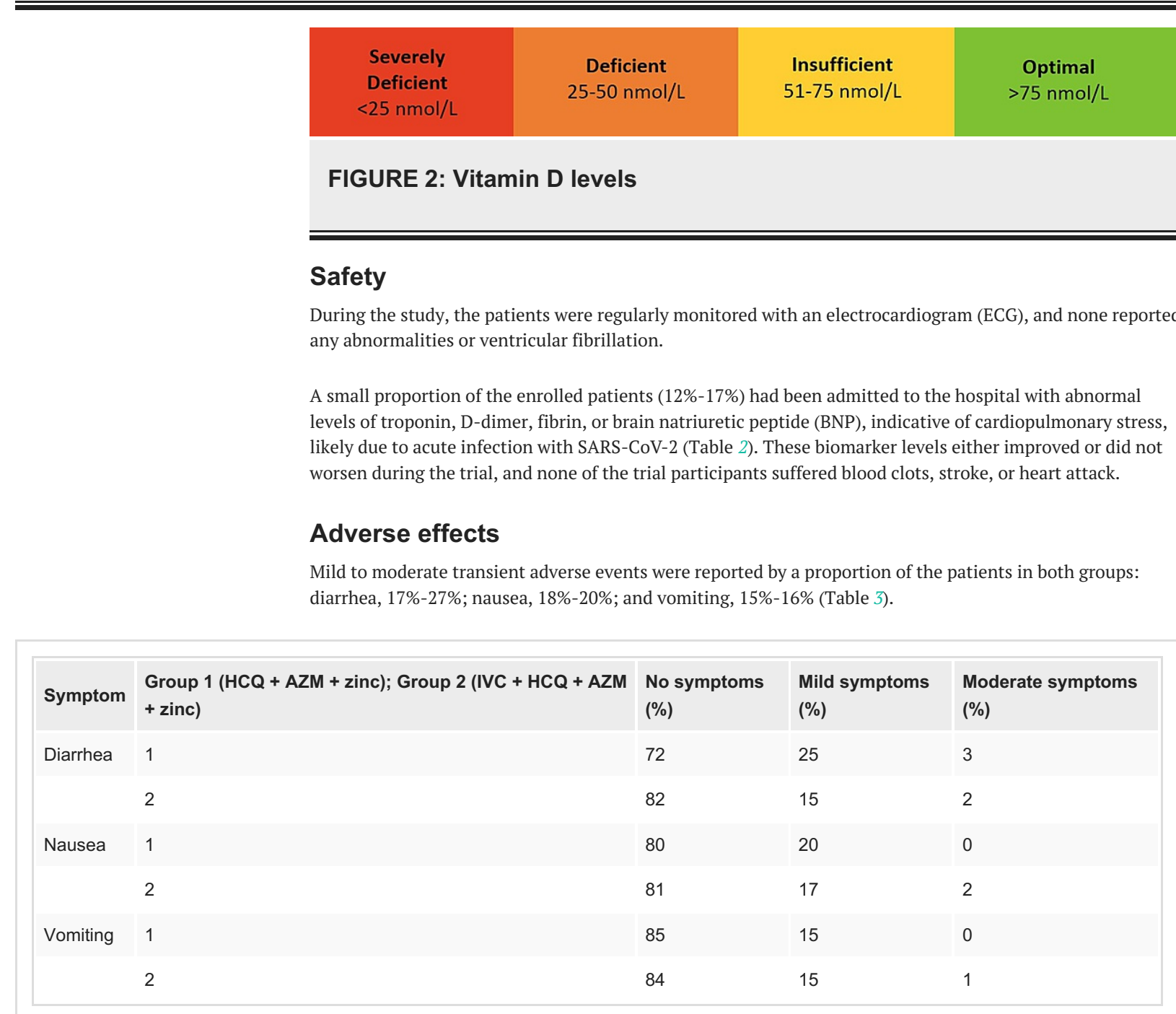

\section{TABLE 3: Adverse effects}

$\mathrm{HCQ}$, hydroxychloroquine; AZM, azithromycin; IVC, intravenous vitamin C

\section{Symptoms on admission}

The enrolled patients experienced the following symptoms on admission into the study: $70 \%$ had difficulty breathing, of which $7 \%$ had severe difficulty breathing and $21 \%$ had moderate difficulties; $60 \%$ had headache ( $56 \%$ mild); $48 \%$ reported cough, of which $11 \%$ had a moderate cough and $3 \%$ had a severe cough; $46 \%$ had a fever, of which the majority were mild (40\%); and $41 \%$ reported a loss of sense of smell.

\section{Key findings}

Almost all hospitalized patients with COVID-19 enrolled in the study were vitamin D deficient (97\%), 55\% were severely vitamin D deficient $(<25 \mathrm{nmol} / \mathrm{L})$ and $42 \%$ were vitamin D deficient $(<50 \mathrm{nmol} / \mathrm{L}) ; 3 \%$ had insufficient vitamin D levels $(<75 \mathrm{nmol} / \mathrm{L})$, and none had optimal vitamin D levels (Table 2$)$.

This finding is in line with the international literature, linking low vitamin D levels with higher susceptibility to symptomatic respiratory infection, including COVID-19.

In our trial, we found a statistically significant correlation between vitamin D levels and ICU admission. The lower the vitamin D level, the higher the probability of being admitted to the ICU $(14.2 \mathrm{nmol} / \mathrm{L}(\mathrm{n}=13)$ versus $25.1 \mathrm{nmol} / \mathrm{L}(\mathrm{n}=224) ; \mathrm{p}<0.0001)$. Furthermore, we found a statistically significant correlation between lower baseline vitamin $\mathrm{D}$ levels and longer hospital stay $(r=-0.195 ; \mathrm{p}=0.003)$. Vitamin $\mathrm{D}$ levels 


\section{Cureus}

were comparable by gender and age.

\section{Outcomes}

All but one patient in our trial fully recovered, half (52\%) of the participants after 15 days, and half (48\%) at 45 days follow-up since enrolment (Table 4).

During the hospital stay, $40 \%$ of the patients required oxygen, and $6 \%$ were admitted to the ICU; one patient was treated on a ventilator, one required renal replacement, and one died (Table 4).

One 70-year-old female patient with heart and lung disease died after 17 days in the ICU and 22 days in the hospital; her vitamin D level was $6 \mathrm{nmol} / \mathrm{L}$ on admission $(<25 \mathrm{nmol} / \mathrm{L}=$ severely deficient $)$.

\begin{tabular}{|c|c|c|}
\hline Variable & $\mathbf{N}(\%)$ & Comment \\
\hline \multicolumn{3}{|l|}{ During hospital stay } \\
\hline Requiring supplemental oxygen & $82(40 \%)$ & \\
\hline ICU admission & $13(6 \%)$ & \\
\hline Ventilator & 1 & \\
\hline Renal replacement & 1 & \\
\hline Died & 1 & Vitamin $\mathrm{D}=6 \mathrm{nmol} / \mathrm{L}$ \\
\hline \multicolumn{3}{|l|}{ Day 15 follow-up } \\
\hline Total recovery/no/mild symptoms & $120(52 \%) / 45(19 \%) / 68(29 \%)$ & \\
\hline \multicolumn{3}{|l|}{ Day 45 follow-up } \\
\hline Total recovery & $236(99.6 \%)$ & \\
\hline
\end{tabular}

\section{TABLE 4: Outcomes}

A larger proportion of participants in group 2, which received additional IV vitamin C therapy, were symptom-free and discharged from the hospital earlier on day 15 compared with day 45 . This difference was statistically significant, indicating that IV vitamin $\mathrm{C}$ treatment contributes significantly to quicker recovery (day 15 versus day $45 ; \mathrm{p}=0.0069$ ) (Table 5).

\begin{tabular}{|c|c|c|c|}
\hline \multirow[b]{2}{*}{ Group } & \multirow[b]{2}{*}{ All N (\%) } & \multicolumn{2}{|c|}{ Total recovery (symptom-free, discharged from the hospital); N (\%) } \\
\hline & & Day 15 & Day 45 \\
\hline $1(H C Q+A Z M+z i n c)$ & $75(32 \%)$ & $29(39 \%)$ & $46(61 \%)$ \\
\hline 2 (IVC + HCQ + AZM + zinc) & $162(68 \%)$ & $93(57 \%)$ & $68(42 \%)$ \\
\hline Total & 237 & $122(51 \%)$ & $114(49 \%)$ \\
\hline
\end{tabular}

\section{TABLE 5: Outcomes by treatment group}

$\mathrm{HCQ}$, hydroxychloroquine; AZM, azithromycin; IVC, intravenous vitamin C

Chi-square statistical analysis: IV vitamin C treatment contributed significantly to quicker recovery (day 15 versus day $45 ; p=0.0069$ )

\section{Discussion}

Our study suggests that the combination of hydroxychloroquine (HCQ), azithromycin (AZM), and zinc with or without IV vitamin C is safe and effective in the early treatment of COVID-19. No cardiac side effects were observed. 
All but one patient $(99.6 \% ; n=236 / 237)$ in our trial fully recovered, with IV vitamin C contributing to a significantly quicker recovery (15 days versus 45 days until discharge).

Our study's findings are in line with the international literature, whereby the treatment of COVID-19 with HCQ, zinc, and AZM or intravenous vitamin $\mathrm{C}$ has shown to be effective in aiding recovery and reducing mortality.

The effectiveness of HCQ with or without AZM in the treatment of COVID-19 has been demonstrated in a meta-analysis of more than 290 trials involving more than 412,000 patients, whereby improvement of symptoms and prevention of death were achieved at $64 \%-75 \%$ if treatment was provided early [6].

The combination of HCQ, AZM, and zinc, also known as the Zelenko protocol, has been shown to reduce hospitalization and mortality significantly, whereby a significantly smaller number of patients in the treatment group was hospitalized or died compared with the untreated group (hospitalized: $3 \%$ treated versus $15 \%$ untreated; died: $0.7 \%$ treated versus $3.4 \%$ untreated) [2].

To date, few studies have looked into the effectiveness of early treatment of patients with COVID-19 with vitamin C/ascorbic acid [22]. One study found that high-dose intravenous vitamin C provided a $72 \%$ improvement in symptoms and reduced recovery time [17]. In contrast, a study that used an oral combination of vitamin $\mathrm{C}$ and zinc did not find a significant difference in the improvement of symptoms between the treatment and the control group [23].

Our study is the first to combine HCQ, AZM, and zinc with high-dose intravenous vitamin C therapy, resulting in the total recovery of $99.6 \%$ of participants, whereby IVC contributed to a significantly quicker recovery and discharge from the hospital. The treatment protocol was highly tolerable and did not cause any cardiac complications.

Importantly, our study confirmed vitamin D deficiency to be a high-risk factor of severe COVID-19 disease and hospitalization, with $97 \%$ of our study's patient cohort being vitamin D deficient, of which $55 \%$ were severely vitamin D deficient.

This finding is in line with the international literature, highlighting the importance of adequate vitamin D levels for immune function, prevention of acute respiratory infections including COVID-19, and recovery $[18,19,24,25]$.

Specifically, vitamin D protects against pathogens including viruses via the innate and adaptive immune system, involving white blood cells and T-cells [21].

Several studies conducted earlier in the pandemic have linked vitamin D deficiency with the risk and severity of COVID-19 infection and hospitalization [26,27], while a recent systematic review and meta-analysis of eight studies and $>1500$ participants concluded that vitamin D levels over $50 \mathrm{nmol} / \mathrm{L}$ can reduce the mortality risk of COVID-19 to zero [28].

It is known that a large proportion of Australians are vitamin D deficient [29]. Research has proven vitamin D supplementation to be a key factor to alleviate vitamin D deficiency and subsequently to prevent the onset and severity of acute respiratory tract infections and reduce morbidity and mortality [20].

Higher daily doses of 5000-10000 IU vitamin D3 orally are considered safe and effective in elevating vitamin D deficiency [19].

Furthermore, our study revealed that lower vitamin D levels were significantly correlated with a higher probability of being admitted to the ICU, leading to a significantly longer hospital stay.

While comorbidities contribute to the risk of hospitalization [30] (three-quarters of our study population had comorbidities), severe vitamin D deficiency (6 nmol/L) was the most probable cause for the death of the 70year-old patient with lung and heart disease.

\section{Conclusions}

In summary, our study found vitamin D deficiency to be a high-risk factor for severe COVID-19 disease and hospitalization, with $97 \%$ of our study's patient cohort being vitamin D deficient, of which $55 \%$ were severely vitamin D deficient, and none had optimal levels. In addition, vitamin D levels were significantly correlated to ICU admission and longer hospital stay.

Furthermore, our study contributes to the evidence of HCQ, AZM, and zinc with or without IV vitamin C being safe and effective in the treatment of COVID-19, with IV vitamin C contributing to a significantly quicker recovery. 
Future research based on the findings in stage 1 of our trial in line with the international literature of the importance of adequate vitamin D levels to immune function and recovery are encouraged to adapt the protocol for the next stage of the trial by adding a high-dose vitamin D3 to all enrolled patients.

\section{Appendices}

\section{Definitions of Adverse Events}

Diarrhea

Grade 1: Increase of < 4 stools per day over baseline; mild increase in ostomy output compared with baseline

Grade 2: Increase of 4-6 stools per day over baseline; moderate increase in ostomy output compared with baseline; limiting instrumental ADL

Grade 3: Increase of $\geqslant 7$ stools per day over baseline; hospitalization indicated; severe increase in ostomy output compared with baseline; limiting self-care

Grade 4: Life-threatening consequences; urgent intervention indicated

Nausea

Grade 1: Loss of appetite without alteration in eating habits

Grade 2: Oral intake decreased without significant weight loss, dehydration, or malnutrition

Grade 3: Inadequate oral caloric or fluid intake; tube feeding, TPN, or hospitalization indicated

Vomiting

Grade 1: Intervention not indicated

Grade 2: Medical intervention indicated

Grade 3: Tube feeding, TPN, or hospitalization indicated

Grade 4: Life-threatening consequences

\section{Additional Information}

\section{Disclosures}

Human subjects: Consent was obtained or waived by all participants in this study. National Institute of Integrative Medicine Human Research Ethics Committee issued approval 0068N 2020. The trial was approved by the National Health and Medical Research Council (NHMRC)-endorsed National Institute of Integrative Medicine (NIIM) Human Research Ethics Committee in Australia, the Turkish Ethics Committees at the Ministry of Health in Turkey, and participating hospitals. Animal subjects: All authors have confirmed that this study did not involve animal subjects or tissue. Conflicts of interest: In compliance with the ICMJE uniform disclosure form, all authors declare the following: Payment/services info: The trial was supported by the Rinehart Medical Foundation (RMF), which provided funding for multicenter trial coordination, trial medication and supplements, testing, and chief investigators, doctors, and staff involved in the trial. The RMF was not involved in the study design, data collection, analysis, and manuscript preparation. Financial relationships: All authors have declared that they have no financial relationships at present or within the previous three years with any organizations that might have an interest in the submitted work. Other relationships: All authors have declared that there are no other relationships or activities that could appear to have influenced the submitted work.

\section{Acknowledgements}

We gratefully acknowledge our collaborators in Turkey, Dr. Cemal Cingi, and Mustafa Akubulut and the Continuous Education and Scientific Research Association (CESRA) in Turkey. We also thankfully acknowledge Dr. Ian Dettman and Biological Therapies who supported this trial by providing sodium ascorbate solutions for the intravenous vitamin $C$ therapy. We are also grateful for the contributions of Dr. Peter Eng and Prof. Ian Brighthope as part of the Trial Advisory Committee.

\section{References}

1. Derwand R, Scholz M: Does zinc supplementation enhance the clinical efficacy of 
chloroquine/hydroxychloroquine to win today's battle against COVID-19?. Med Hypotheses. 2020, 142:109815. 10.1016/j.mehy.2020.109815

2. Derwand R, Scholz M, Zelenko V: COVID-19 outpatients: early risk-stratified treatment with zinc plus lowdose hydroxychloroquine and azithromycin: a retrospective case series study. Int J Antimicrob Agents. 2020, 56:106214. 10.1016/j.ijantimicag.2020.106214

3. Yang N, Shen HM: Targeting the endocytic pathway and autophagy process as a novel therapeutic strategy in COVID-19. Int J Biol Sci. 2020, 16:1724-31. 10.7150/ijbs.45498

4. Hu TY, Frieman M, Wolfram J: Insights from nanomedicine into chloroquine efficacy against COVID-19 . Nat Nanotechnol. 2020, 15:247-9. 10.1038/s41565-020-0674-9

5. Gautret P, Lagier JC, Parola P, et al.: Hydroxychloroquine and azithromycin as a treatment of COVID-19: results of an open-label non-randomized clinical trial. Int J Antimicrob Agents. 2020, 56:105949. 10.1016/j.ijantimicag.2020.105949

6. HCQ for COVID- 19: real-time meta analysis of 294 studies . (2021). https://hcqmeta.com/.

7. Bosseboeuf E, Aubry M, Nhan T, de Pina JJ, Rolain JM, Raoult D, Musso D: Azithromycin inhibits the replication of Zika virus. J Antivir Antiretrovir. 2018, 10:6-11. 10.4172/1948-5964.1000173

8. Retallack H, Di Lullo E, Arias C, et al.: Zika virus cell tropism in the developing human brain and inhibition by azithromycin. PNAS. 2016, 113:14408-13. 10.1073/pnas.1618029113/-/DCSupplemental

9. Andreani J, Le Bideau M, Duflot I, et al.: In vitro testing of combined hydroxychloroquine and azithromycin on SARS-CoV-2 shows synergistic effect. Microb Pathog. 2020, 145:104228. 10.1016/j.micpath.2020.104228

10. Tanriverdİ E, Çörtük M, Yildirim BZ, et al.: Hydroxychloroquine plus azithromycin and early hospital admission are beneficial in COVID-19 patients: Turkish experience with real-life data. Turk J Med Sci. 2021, 51:10-5. 10.3906/sag-2005-82

11. Xue J, Moyer A, Peng B, Wu J, Hannafon BN, Ding WQ: Chloroquine is a zinc ionophore. PLoS One. 2014, 9:e109180. 10.1371/journal.pone.0109180

12. Read SA, Obeid S, Ahlenstiel C, Ahlenstiel G: The role of zinc in antiviral immunity. Adv Nutr. 2019, 10:696710. 10.1093/advances/nmz013

13. te Velthuis AJ, van den Worm SH, Sims AC, Baric RS, Snijder EJ, van Hemert MJ: Zn(2+) inhibits coronavirus and arterivirus RNA polymerase activity in vitro and zinc ionophores block the replication of these viruses in cell culture. PLoS Pathog. 2010, 6:e1001176. 10.1371/journal.ppat.1001176

14. Cerullo G, Negro M, Parimbelli M, et al.: The long history of vitamin C: from prevention of the common cold to potential aid in the treatment of COVID-19. Front Immunol. 2020, 11:574029. 10.3389/fimmu.2020.574029

15. Mahmoodpoor A, Shadvar K, Sanaie S, Hadipoor MR, Pourmoghaddam MA, Saghaleini SH: Effect of vitamin $\mathrm{C}$ on mortality of critically ill patients with severe pneumonia in intensive care unit: a preliminary study. BMC Infect Dis. 2021, 21:616. 10.1186/s12879-021-06288-0

16. Huang L, Wang L, Tan J, Liu H, Ni Y: High-dose vitamin C intravenous infusion in the treatment of patients with COVID-19: a protocol for systematic review and meta-analysis. Medicine (Baltimore). 2021, 100:e25876. 10.1097/MD.0000000000025876

17. Zhao B, Liu M, Liu P, et al.: High dose intravenous vitamin C for preventing the disease aggravation of moderate COVID-19 pneumonia. a retrospective propensity matched before-after study. Front Pharmacol. 2021, 12:638556. 10.3389/fphar.2021.638556

18. Martineau AR, Jolliffe DA, Hooper RL, et al.: Vitamin D supplementation to prevent acute respiratory tract infections: systematic review and meta-analysis of individual participant data. BMJ. 2017, 356:i6583. 10.1136/bmj.i6583

19. Benskin LL: A basic review of the preliminary evidence that COVID-19 risk and severity is increased in vitamin D deficiency. Front Public Health. 2020, 8:513. 10.3389/fpubh.2020.00513

20. Brighthope I, Sali Am A, Ried K: Vitamin-D and COVID-19: time for the profession to take a stand. Adv Integr Med. 2021, 8:77-8. 10.1016/j.aimed.2021.01.003

21. Xu Y, Baylink DJ, Chen CS, et al.: The importance of vitamin d metabolism as a potential prophylactic, immunoregulatory and neuroprotective treatment for COVID-19. J Transl Med. 2020, 18:322. 10.1186/s12967-020-02488-5

22. Meta-analysis of vitamin C Covid-19 treatment studies . (2021). https://c19vitaminc.com/.

23. Thomas S, Patel D, Bittel B, et al.: Effect of high-dose zinc and ascorbic acid supplementation vs usual care on symptom length and reduction among ambulatory patients with SARS-CoV-2 infection: the COVID A to Z randomized clinical trial. JAMA Netw Open. 2021, 4:e210369. 10.1001/jamanetworkopen.2021.0369

24. Yildiz M, Senel MU, Kavurgaci S, Ozturk FE, Ozturk A: The prognostic significance of vitamin D deficiency in patients with COVID-19 pneumonia. Bratisl Lek Listy. 2021, 122:744-7. 10.4149/BLL_2021_119

25. Matin S, Fouladi N, Pahlevan Y, et al.: The sufficient vitamin D and albumin level have a protective effect on COVID-19 infection. Arch Microbiol. 2021, 203:5153-62. 10.1007/s00203-021-02482-5

26. Merzon E, Tworowski D, Gorohovski A, Vinker S, Golan Cohen A, Green I, Frenkel-Morgenstern M: Low plasma 25(OH) vitamin D level is associated with increased risk of COVID-19 infection: an Israeli population-based study. FEBS J. 2020, 287:3693-702. 10.1111/febs.15495

27. Hernández JL, Nan D, Fernandez-Ayala M, et al.: Vitamin D status in hospitalized patients with SARS-CoV2 infection. J Clin Endocrinol Metab. 2021, 106:e1343-53. 10.1210/clinem/dgaa733

28. Borsche L, Glauner B, Mendel Jv: COVID-19 mortality risk correlates inversely with vitamin D3 status, and a mortality rate close to zero could theoretically be achieved at $50 \mathrm{ng} / \mathrm{ml} 25(\mathrm{OH}) \mathrm{D} 3$ : results of a systematic review and meta-analysis. Nutrients. 2021, 13:3596. 10.1101/2021.09.22.21263977

29. Travica N, Ried K, Bujnowski R, Sali A: Integrative health check reveals suboptimal levels in a number of vital biomarkers. Adv Integr Med. 2015, 2:135-40. 10.1016/j.aimed.2015.11.002

30. Baradaran A, Ebrahimzadeh MH, Baradaran A, Kachooei AR: Prevalence of comorbidities in COVID-19 patients: a systematic review and meta-analysis. Arch Bone Jt Surg. 2020, 8:247-55.

10.22038/abjs.2020.47754.2346 